\title{
COMPRESSED SENSING SYSTEM FOR EFFICIENT ECG SIGNAL COMPRESSION
}

\author{
Lakshana Nagaraj $^{1}$, M.Varshini ${ }^{2}$, Manikandan K.R ${ }^{3}$, B. Sreenesh ${ }^{4}$, V.Sivakumar ${ }^{5}$, S.Veni $^{6}$ \\ ${ }^{I}$ Student, Department of ECE, Amrita School of Engineering, Amrita Vishwa Vidya Peetham, Amrita Nagar, Coimbatore - \\ 641112, Tamil Nadu, India \\ ${ }^{2}$ Student, Department of ECE, Amrita School of Engineering, Amrita Vishwa Vidya Peetham, Amrita Nagar, Coimbatore - \\ 641112, Tamil Nadu, India \\ ${ }^{3}$ Student, Department of ECE, Amrita School of Engineering, Amrita Vishwa Vidya Peetham, Amrita Nagar, Coimbatore - \\ 641112, Tamil Nadu, India \\ ${ }^{4}$ Student, Department of ECE, Amrita School of Engineering, Amrita Vishwa Vidya Peetham, Amrita Nagar, Coimbatore - \\ 641112, Tamil Nadu, India \\ ${ }^{5}$ Student, Department of ECE, Amrita School of Engineering, Amrita Vishwa Vidya Peetham, Amrita Nagar, Coimbatore - \\ 641112, Tamil Nadu, India \\ ${ }^{6}$ Assoc. Professor, Department of ECE, Amrita School of Engineering, Amrita Vishwa Vidya Peetham, Amrita Nagar, \\ Coimbatore - 641112, Tamil Nadu, India
}

\section{Abstract}

Compressed sensing (CS) $[1,13,14]$ is a novel idea wherein a signal can be sampled at sub-Nyquist rates and still be effectively reconstructed. Many natural signals such as the ECG signal are sparse and have sparse representation when expressed in a suitable basis. Compressed sensing exploits the sparsity by acquiring a small number of projections on to random vectors which are sufficient to recover the signal. This theory enables an effective implementation of patient-centric telecardiology or mobile cardiology systems. It guarantees improvement in performance by drastically reducing the number of samples that requires to be either stored or transmitted. Specifically in the context of ECG monitoring systems, CS signal acquisition revolutionizes miniaturization of the hardware and improves its energy efficiency. This work proposes to create a Compressed sensing based mathematical framework for the acquisition and recovery of an ECG signal which has been further classified into normal and abnormal categories. During the compression stage, a sensing matrix which results in a low PRD (Percentage root mean square distortion) is used and an efficient reconstruction algorithm is employed to retrieve the most valuable information from the ECG signal. The classification of the ECG signal is done by studying the region of interest i.e. the QRS complex. The heart rate, the interval between two $R$ peaks and the amplitude of the R peaks contributes chiefly to determining an abnormality in the ECG signal. This model can be implemented with a wireless body sensor network (WBSN) which may be used to alert a doctor in emergency situations.

Keywords: Compressed Sensing, Electrocardiogram, Classification

\section{INTRODUCTION}

According to the World Health Organization (WHO), an estimate of 17 million people globally die of cardiovascular diseases (CVD) [10]. Sensors in the pads of the electrodes attached to the body detect the electrical activity of the heart. A portable body sensor carried by the patients will record and store their ECG signals for long periods of time (up to two weeks). Hence compression of ECG signals is vital in order to minimize computational complexity in terms of storage and power requirements. For the purpose of this paper, the ECG signals have been obtained from the MIT-BIH Arrhythmia Database [16].

According to previous research, Mamaghanian et. al. [3] proposes implementation of compressed sensing signal acquisition on the state-of-the-art Shimmer WBSN mote using a uniform binary sensing matrix. The major drawback here is that the selectively spread information of ECG is not been taken into account. This is rectified by a non uniform binary sensing matrix [5] wherein more information is acquired and preserved from the region of interest (ROI) i.e. QRS complex. In this paper, we further detect if the patient is suffering from an arrhythmia. The objective of this paper is to exploit the additional information reserved from the region of interest to accurately predict arrhythmia with less data.

The portable body sensor is required to be light-weight and miniaturized for the patient's convenience. Thus using compressed sensing $[1,13$, and 14$]$ while sampling the ECG signal enables to accomplish the above requirements. The recovery of the compressed signal can be performed on an embedded platform such as a smart phone enabling the doctor 
to obtain immediate notification in case the ECG signal is abnormal.

The ECG signal has been further classified into normal and abnormal categories. At the receiver end, the reconstructed ECG signal is categorized as abnormal based on a feature extraction technique. This procedure is operated in a remote location by a local server which handles the more complex mathematical calculations. The performance of compression algorithms is usually quantified and assessed in terms of two performance metrics [3]:

1. Percentage root mean square distortion (PRD): is defined as

$$
P R D=\frac{\left\|x_{\text {orig }}-x_{\text {rec }}\right\|}{\left\|x_{\text {orig }}\right\|} \times 100
$$

Where $x_{\text {orig }}$ represents the original signal vector $\mathrm{x}$ and $x_{\text {rec }}$ represents the reconstructed signal.

2. Compression Ratio (CR) : is defined as

$C R=\frac{b_{\text {orig }}-b_{\text {comp }}}{b_{\text {orig }}} \times 100$

Where $b_{\text {orig }}$ represents the number of bits required for the original signal and $b_{\text {comp }}$ represents the number of bits required for the reconstructed signal.

This paper has the following parts. In section II, the theory of compressed sensing is reviewed briefly. In section III, the methodology followed to compress and reconstruct an ECG signal has been discussed followed by section IV where the classification of the ECG signal is described. The simulation results and final conclusions are presented in section V and VI respectively.

\section{COMPRESSED SENSING- A REVIEW}

The general approach to sample a signal follows the nyquistshannon's theorem: the sampling rate must be at least twice the maximum frequency present in the signal [17]. Compressive sensing $[1,13$, and 14] acquires a signal by just capturing a small number of indirect measurements. This makes it highly effective. For this methodology to succeed, the signal must be sparse. To be precise, it must have minimal non zero coefficients, represented as $\alpha(\mathrm{N} \times 1$ column vector of weighting) coefficients when expressed with a set of orthonormal basis $\Psi$ i.e.

$$
x=\sum_{i=1}^{n} \alpha_{i} \varphi_{i}=\varphi \alpha
$$

Given $\mathrm{x} \epsilon R^{n}$ and suppose $\mathrm{x}$ is $\mathrm{r}$-sparse in basis $\Psi$, then selecting $M$ randomly projected measurements using the sensing matrix such that,

$$
M>r \log \frac{N}{r}
$$

Would ensure the exact reconstruction of the signal $\mathrm{x}$ with overwhelming probability. In compressed sensing method, linear superposition of $\mathrm{x}$ samples is obtained. $\mathrm{y}$, the output frame is a vector of $\mathrm{M}$ linear projections of $\mathrm{x}$ onto another basis $\Phi(\mathrm{M}<\mathrm{N})$

$$
y=\Phi x=\Psi \Phi \alpha
$$

Where $\Phi$ represents the sensing matrix. The original signal $\mathrm{x}$ is reconstructed by solving the convex minimization problem that follows

$$
\min \mid "-\cdots " \text { Fig -1: } \text {;ubject to } \Psi \Phi \alpha=y
$$

\section{COMPRESSED SENSING MODULES FOR ECG SIGNAL COMPRESSION}

The first module modeled in the compressed sensing system involves choosing an effective sensing matrix to compress the signal. A sensing matrix of size $\mathrm{M} \times \mathrm{N}$ is multiplied with the original ECG data of size $N \times 1$ to effectively retain the necessary information in less number of samples thus resulting in the compressed signal $(\mathrm{M} \times 1)$. In order to acquire and preserve more information from the QRS complex in each heartbeat, we use a sensing matrix which obtains more information from this region of interest (ROI). The non uniform binary sensing matrix achieves the above criterion effectively [5]. Fig.1 describes the block diagram of the compressed sensing modules used in this work.

The non-uniform binary sensing matrix $[\Phi]$ is dependent on the ECG signal to be sampled. It consists of two uniform binary sensing matrices where all of its entries belong to the binary set and the probability distribution of its entries is i.i.d Bernoulli. In the algorithm followed, the location of QRS complex is first detected and the distance between the first Q and $\mathrm{S}$ peaks is determined. Using this as the window size $(\mathrm{W})$, the first binary sensing matrix $(\mathrm{M} 1 \times(\mathrm{N}-\mathrm{W}))$ is horizontally shifted over the second binary sensing matrix $(\mathrm{M} 2 \times \mathrm{N})$ to be aligned with the $\mathrm{R}$ peak., where $\mathrm{M} 1+\mathrm{M} 2=\mathrm{M}$. 


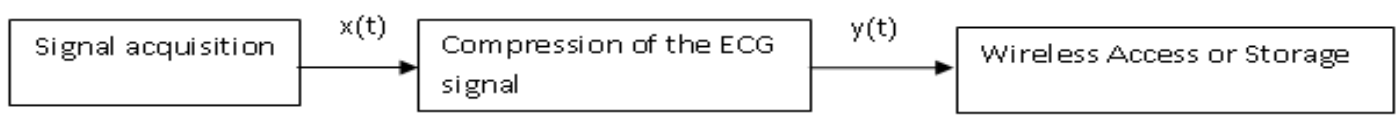

(a)

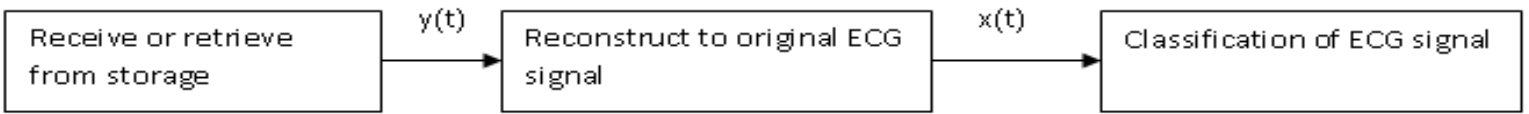

(b)

Figure 1. (a) Block diagram for ECG signal compression, (b) Block diagram for the retrieval and reconstruction of the ECG signal

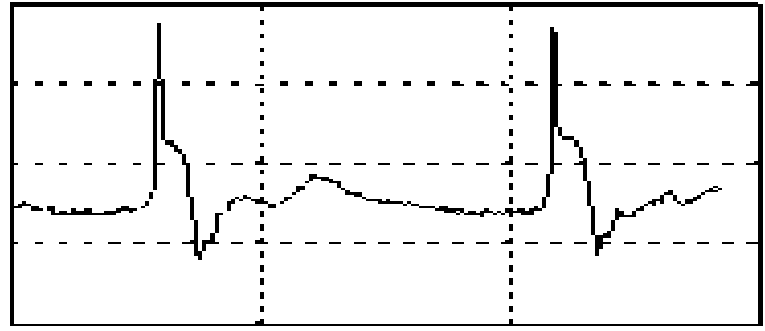

(a)

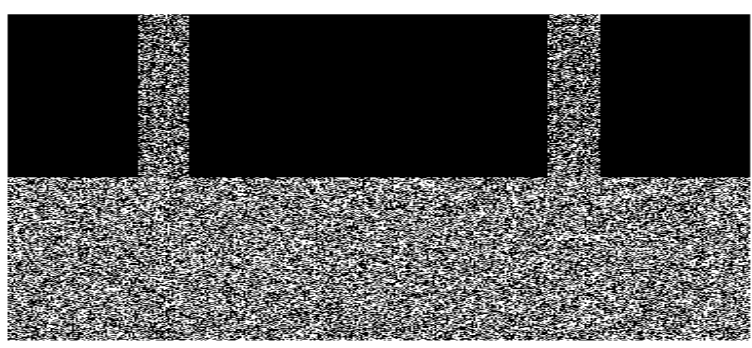

(b)

Fig -2: (a) Original ECG signal, (b) Non-uniform binary matrix

The original signal is recovered from its compressed state using convex optimization algorithms. Minimizing the L1 norm results in the sparsest solution in the appropriate domain. The signal is reconstructed using A*OMP algorithm [9], where the solution is searched on several paths of a search tree. It outperforms the conventional CS reconstruction methods namely Basis Pursuit (BP), Orthogonal Matching Pursuit (OMP), and Subspace Pursuit (SP).

\section{CLASSIFICATION OF ECG SIGNAL}

The necessity to classify an ECG signal becomes vital in assisting which of the compressed data needs to be reconstructed for a cardiologist to diagnose the disease. A patient's heart condition is broadly classified as normal and abnormal. To diagnose any cardiac arrhythmia, the stored compressed measurements is reconstructed to original ECG signal through the algorithm A*OMP [9]. The region of interest shows the rapid depolarization in the right and the left ventricles, thus allowing the ECG to record the electrical potential generated. Cardiac arrhythmia is the condition in which the heart's sinus rhythm is interrupted. The heart rhythm is typically monitored by ECG where the QRS complex, associated with the excitation of ventricles plays an important role to identify cardiac arrhythmias. The major abnormal situations include irregular peak value, irregular distance between peaks of ECG signal and varying range of QRS complex beyond its estimated span.

The distance between adjacent QRS complexes, RR interval, determines the rhythm of heart rate i.e. regular or irregular. The interval between two $\mathrm{R}$ peaks has duration of 0.6 to 1.2 seconds. If the rhythm is irregular, it is commonly linked with atrial flutter and atrial fibrillation. The normal resting heart rate is between 50-100 bpm. The QRS complex has duration of 80-120 ms, if the QRS complex widens beyond this range it could trigger hypokalemia which may occasionally provoke cardiac arrhythmias. Also the amplitude of $\mathrm{R}$ peak measured from the V5 lead (MIT-BIH ECG database [16]) normally lies below a value of $2.6 \mathrm{mV}$. The ECG signal has been classified as abnormal when any one of the above parameters exceeds their range.

\section{RESULTS AND DISCUSSION}

As depicted in fig.1, the received signal $\mathrm{y}$ is obtained and reconstructed using $\mathrm{A}^{*} \mathrm{OMP}$ algorithm, where the solution is searched on several paths of a search tree. This follows multipath search strategy that combines first best search and OMP. This algorithm is completely general to all sparse signals and aims to find a closer result to the true $l_{0}$ solution, thus improving reconstruction quality. The receiver end is equipped with a multifaceted system which is capable of handling complex iterative operations with ease. Following the reconstruction, the recovered signal is further classified into normal or abnormal based on the R-R interval. 

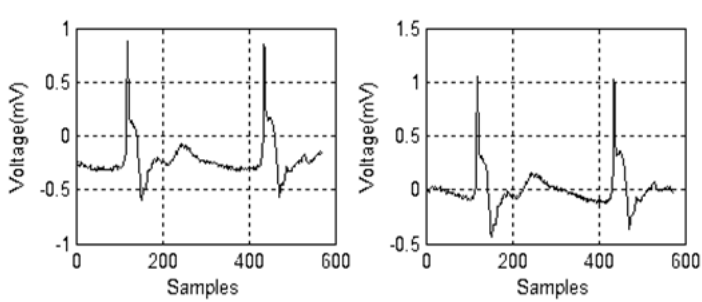

Fig -3: (Left) Original ECG signal, (Right) Detrended ECG signal

The R-R interval between each QRS complex is calculated from the reconstructed signal. When this interval does not lie in the range of $600 \mathrm{~ms}-1200 \mathrm{~ms}$, the signal is classified as abnormal. This roughly translates to a normal heart rate of 50 beats per minute (bpm) to $100 \mathrm{bpm}$. When the R-R intervals are calculated from the reconstructed signal the difference is found to be as small as $3 \mathrm{~ms}$ from the original signal, which is just a difference of a single sample of the ECG signal. Hence, the classification of ECG signal is not compromised. The results are verified for various compression ratios, as shown in Fig.5.
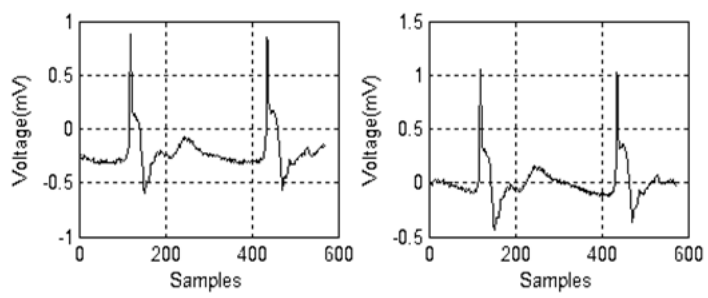

Fig -4: Detection of QRS peaks

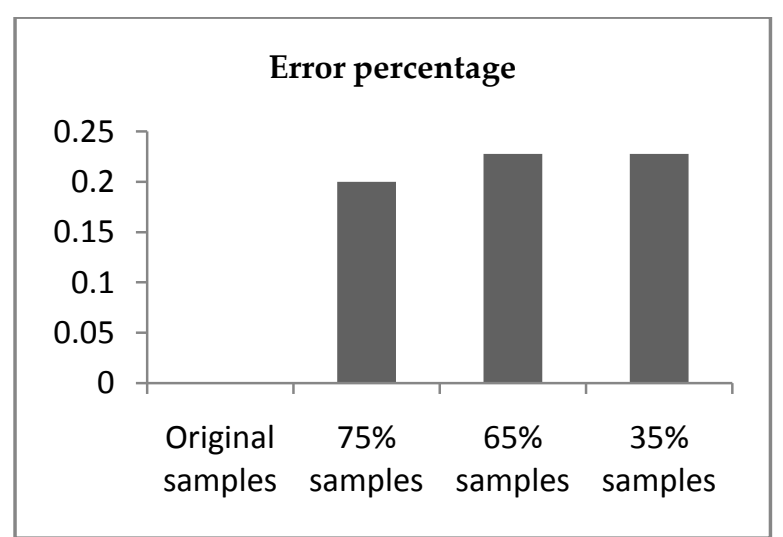

Fig -5: Error percentage of detection of R-R interval for various compression ratios

\section{CONCLUSIONS}

A compressed sensing model for ECG signal compression using non-uniform binary matrix is employed in this work. In addition, classification of ECG signal is performed after the ECG signal is acquired using the new compressive sensing paradigm and has been reconstructed using convex optimization algorithms. The use of non-uniform binary matrix ensures preservation of data in the region of interest (ROI) namely the QRS complex. This results in high efficiency while calculation of R-R intervals to predict the arrhythmia conditions of the patient. This is highly advantageous in implementation of WBSN system for monitoring of patients who require critical care and constant observation.

\section{REFERENCES}

[1] D. L. Donoho, "Compressed sensing," IEEE Trans. Inf. Theory, vol.52, no. 4, pp. 1289-1306, 2006.

[2] Hao Ding, Hong Sun, Kun-mean Hou, "Abnormal ECG signal detection based on Compressed Sampling in Wearable ECG sensor," in Proc. Int. Conf. Wireless Communications and Signal Processing (WCSP), Nov. 2011.

[3] H. Mamaghanian, N. Khaled, D. Atienza, and P. Vandergheynst, "Compressed Sensing for Real-Time Energy-Efficient ECG Compression on Wireless Body Sensor Nodes," IEEE International Symposium on Circuits and Systems (ISCAS), 2011.

[4] Anna M. R. Dixon, Emily G. Allstot, Daibashish Gangopadhyay, David J. Allstot, "Compressed Sensing System Considerations for ECG and EMG Wireless Biosensors," IEEE Trans. Biomedical Circuits and Systems, vol. 6, no. 2, April 2012.

[5] Fahimeh Ansari-Ram, Saied Hosseini-Khayat, "ECG Signal Compression Using Compressed Sensing with Nonuniform Binary Matrices," 16th CSI International Symposium, Artificial Intelligence and Signal Processing (AISP 2012).

[6] Thong T. Do, Lu Gan, Nam H. Nguyen, Trac D. Tran, "Fast and Efficient Compressive Sensing using Structurally Random Matrices," IEEE Trans. signal processing, vol. 60, no. 1, Jan. 2012.

[7] Ramin Zahedi , Lucas W. Krakow, Edwin K. P. Chong, Ali Pezeshki, "Adaptive Estimation of Time-Varying Sparse Signals," IEEE Access, vol. 1, pp. 449-464, 2013.

[8] HolgerRauhut, Karin Schnass and Pierre Vandergheynst, "Compressed Sensing and Redundant Dictionaries," IEEE Trans. Inf. Theory, vol. 54, no. 5, pp. 2210-2219, 2008.

[9] Nazim Burak Karahanoglu, Hakan Erdogan, "Compressed sensing signal recovery via $\mathrm{A}^{*}$ orthogonal matching pursuit," IEEE Conference on Acoustics, Speech and Signal Processing (ICASSP), 2011. 
[10] World Health Organization, "Cardiovascular diseases," 2009.

[Online].

Available:http://www.who.int/topics/cardiovascular diseases.

[11] E. G. Allstot, A. Y. Chen, A. M.R. Dixon, D. Gangopadhyay, and D. J. Allstot1, "Compressive Sampling of ECG Bio-Signals: Quantization Noise and Sparsity Considerations," IEEE Biomedical Circuits and Systems Conference (BioCAS), 2010.

[12] L. F. Polania, R. E. Carrillo, M. Blanco-Velasco and K. E. Barner, "Compressed Sensing Based Method for ECG Compression,"IEEE International Conference on Acoustics, Speech and Signal Processing (ICASSP), 2011.

[13] E. J. Candès, and M. B. Wakin, "An Introduction To Compressive Sampling," IEEE Signal Proc Mag., 25: 21-30, 2008.

[14] R. G. Baraniuk, "Compressive Sensing," IEEE Signal Proc Mag., 24: 118-120, 2007.

[15] E. Candès, J. Romberg, and T. Tao, "Robust uncertainty principles: Exact signal reconstruction from highly incomplete frequency information," IEEE Trans. Inform. Theory, vol. 52, no. 2, pp. 489-509, Feb. 2006.

[16] MIT-BIH arrhythmia database. (2005). [online]. Available:http://www.physionet.org/physiobank/databa se/mitdb/

[17] Available:en.wikipedia.org/wiki/Talk:NyquistShannon_sampling_theorem/Archive_1

\section{BIOGRAPHIES}

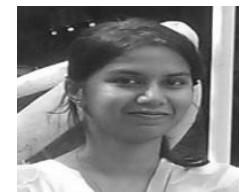

M. Varshini, final year student of Bachelor of Technology in Electronics and Communication Engineering in Amrita School Of Engineering, Coimbatore.

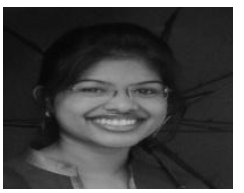

Lakshana Nagaraj, final year student of Bachelor of Technology in Electronics and Communication Engineering in Amrita School Of Engineering, Coimbatore.

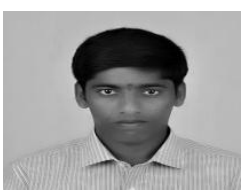

Sivakumar V., final year student of Bachelor of Technology in Electronics and Communication Engineering in Amrita School Of Engineering, Coimbatore.

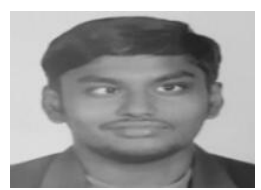

Manikandan K.R., final year student of Bachelor of Technology in Electronics and Communication Engineering in Amrita School Of Engineering, Coimbatore.

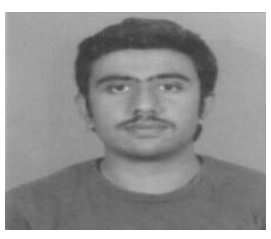

Sreenesh B., final year student of Bachelor of Technology in Electronics and Communication Engineering in Amrita School Of Engineering, Coimbatore.

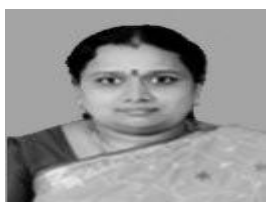

Dr. S.Veni, associate Professor in the department of Electronics and Communication Engineering in Amrita School of Engineering, Coimbatore. 